\title{
Quality of traffic flow on urban arterial streets and its relationship with safety
}

\author{
Vinayak V. Dixit , Anurag Pande Mohamed Abdel-Aty , Abhishek Das , Essam Radwan
}

\begin{abstract}
A B S T R A C T
The two-fluid model for vehicular traffic flow explains the traffic on arterials as a mix of stopped and running vehicles. It describes the relationship between the vehicles' running speed and the fraction of running vehicles. The two parameters of the model essentially represent 'free flow' travel time and level of interaction among vehicles, and may be used to evaluate urban roadway networks and urban corridors with partially limited access. These parameters are influenced by not only the roadway characteristics but also by behavioral aspects of driver population, e.g., aggressiveness. Two-fluid models are estimated for eight arterial corridors in Orlando, FL for this study. The parameters of the two-fluid model were used to evaluate corridor level operations and the correlations of these parameters' with rates of crashes having different types/severity. Significant correlations were found between two-fluid parameters and rear-end and angle crash rates. Rate of severe crashes was also found to be significantly correlated with the model parameter signifying inter-vehicle interactions. While there is need for further analysis, the findings suggest that the two-fluid model parameters may have potential as surrogate measures for traffic safety on urban arterial streets.
\end{abstract}

\section{Introduction}

The two-fluid model developed by Prigogine and Herman (1971) has been used to characterize quality of traffic flow on an urban network. Recent work by Jones and Farhat (2004) demonstrated the validity of the two-fluid model on individual urban arterials and argued that the model provides two performance measures for describing the traffic quality on urban streets (other than freeways). These performance measures are provided in the form of parameters of a curvilinear equation relating stop-time and travel-time per unit distance. The ability of two-fluid model to characterize urban traffic not just at a network level but also at arterial street level was demonstrated using corresponding data from two arterials (Jones and Farhat, 2004). In spite of this renewed attention very few studies have attempted to understand two-fluid model parameters' relationship with driver behavior and road geometry in the context of urban streets.

This research attempts to evaluate corridor level operations using the parameters of the two-fluid model for arterial corridors (which do not include uninterrupted flow facilities such as interstate highways, expressways and freeways), and relate these parameters with geometric factors, aggregate traffic parameters of the arterials (e.g., ADT) and crash rates. The inferences drawn from the analysis presented herein bring to light interesting correlations between the two-fluid model parameters and crash rates (for crashes with varying severity and type), indicating that these parameters may have some potential as safety surrogates on arterial streets.

\section{Background}

\subsection{Two-fluid model and the ergodic assumption}

The two-fluid model assumes that vehicular traffic flow in an urban network or street can be understood as consisting of stopped and running vehicles. The model describes the relationship between the vehicles' running speed (inverse of the time spent running per mile) $\left(v_{r}\right)$ and the fraction of running vehicles $\left(f_{r}\right)$.

$$
v_{r}=v_{m}\left(f_{r}\right)^{n}
$$

where $v_{r}$ is the running speed of the vehicles; $f_{r}$ is the fraction of running vehicles; $v_{m}$ is the maximum speed (corresponding to minimum travel time); $n$ represents one of the two-fluid model parameters.

In general, the subscript ' $r$ ' indicates that the variable is measured for vehicles that are running and the subscript ' $s$ ' indicates that the variable is measured for vehicles that are stopped, subscript ' $m$ ' is used for the maximum speed or minimum travel time per mile. 
When all vehicles are running (i.e., there are no stopped vehicles in the network, $\left.f_{r}=1\right)$, therefore the running speed $\left(v_{r}\right)$ is equal to $v_{m}$. Therefore $v_{m}$ is defined as the average maximum speed, when no vehicles are stopped.

$T_{m}=\frac{1}{v_{m}} \quad\left(T_{m}\right.$ is then the minimum free flow travel time per mile)

Ardekani (1984), through field experiments, showed that it was possible to characterize urban networks by the two-fluid model. The study also used aerial photographs to validate the ergodic assumption of the two-fluid model. The ergodic assumption states that the ratio of running time per mile $\left(T_{r}\right)$ to the travel time per mile $(T)$ is equal to the ratio of the number of vehicles running to the total number of vehicles (Eq. (2)).

$f_{r}=\left(\frac{T_{r}}{T}\right)$

Utilizing Eqs. (1) and (2) and that the running speed is equal to the inverse of the running time per mile.

$T_{r}=T_{m}^{1 / n+1} T^{1 / n+1}$

Eq. (3) describes the relationship between the average travel time per mile $(T)$ and the running time per mile $\left(T_{r}\right) . n$ and $T_{m}$ are parameters of the two-fluid model and determine the sensitivity of the travel time per mile to the stopped time per mile. These parameters have been used in literature to describe the quality of traffic on the networks (Ardekani, 1984). The travel time per mile is defined as the sum of the running time per mile and the stopped time per mile $\left(T_{S}\right)$. Hence Eq. (3) can be rewritten as:

$T_{S}=T-T_{m}^{1 / n+1} T^{1 / n+1}$

when the stopped time per mile diminishes to zero, the travel time per mile is equal to the running time per mile $\left(T=T_{r}\right)$. Replacing $T=T_{r}$ in Eq. (3) results in the travel time per mile being equal to $T_{m}$. Therefore, $T_{m}$ could be considered as the free-flow travel time per mile when there are no stops.

\subsection{Experiments with the two-fluid model: network performance}

Mahmassani et al. (1984) and Williams et al. (1985), using computer simulation based on car following theory, replicated the two fluid models on simple grid networks.

Ardekani (1984) developed two-fluid models for urban traffic networks for various cities (Fig. 1) by collecting data using the chase car methodology. In the chase car methodology the driver is instructed to follow a randomly selected vehicle until it either parks or leaves the designated network. Based on the models it was inferred that Matamoros was the worst performing network, since the model for Matamoros had the highest $y$-intercept (i.e., $T_{m}$ ) and the steepest slope (i.e., $n$ ). The inference was based on the idea that higher $n$, suggested that the travel time increases at a faster rate as the stopped time increases, indicating that the network deteriorates rapidly during congestion. Also, the lower the value of $T_{m}$ meant higher free-flow speeds in un-congested conditions. Based on this, Austin can be considered to be the best performing network.

Recently, Vo et al. (2007) conducted a before-after (1994 vs. 2003) study of the Arlington, Texas network. They observed that there has been no significant change in the performance of the network despite large number of projects to improve the network. They conducted similar analysis for the Dallas and Fort-worth networks. Ayadh (1986), Ardekani et al. (1992), and Bhat (1994) selected various network features and estimated regression models to understand the effects of these network features on $T_{m}$ and $n$. Table 1 summarizes the sign of correlation between the network features and the two parameters of the two fluid model, i.e., $T_{m}$ and $n$. "+" sign signifies a positive correlation between the factor and

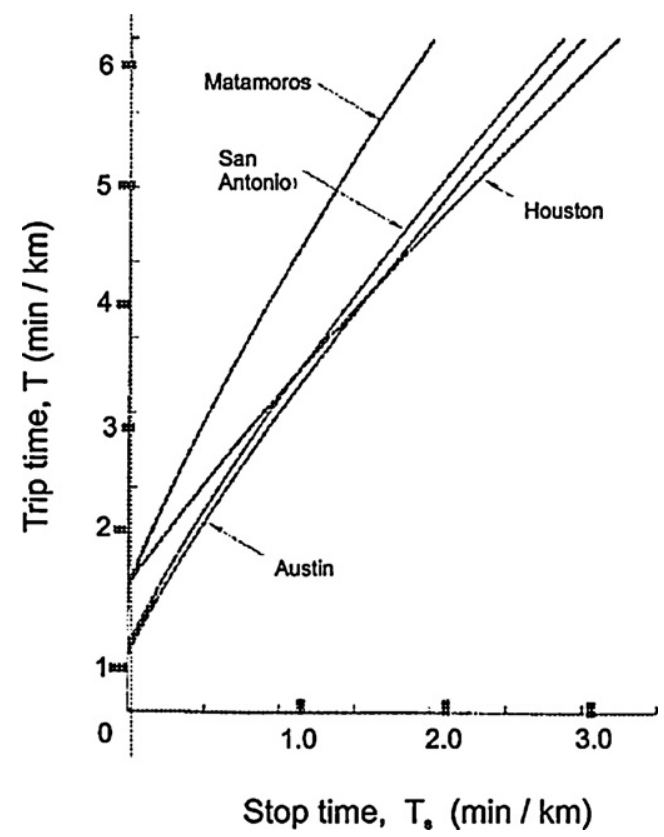

Fig. 1. Two-fluid model for various cities (Herman and Ardekani, 1984).

parameter and “-" sign signifies a negative correlation. For example, an increase in signal density would increase $T_{m}$ (depicted with a "+" sign) and is negatively associated with value of $n$ (depicted with a "-" sign).

\subsection{Experiments with the two-fluid model: driver behavior}

The parameters of two-fluid model have also been used to characterize driver behavior. Herman et al. (1988) studied the effects of extreme driver behaviors on the two-fluid model. They found that a test car driver instructed to drive aggressively in a network established a significantly different individual two-fluid trend than one instructed to drive conservatively in the same network at the same time. In their study, data were also collected using chasecar method by following randomly sampled vehicles driving in the network. The two fluid model generated from following randomly selected vehicles in the network was considered to be 'normal' or representative of the overall network. As expected, aggressive drivers were found to have a lower $T_{m}$ than normal drivers and normal drivers had lower $T_{m}$ than conservative drivers. They also found that all three two-fluid trends (aggressive, conservative, and normal) converged as the stopped time per mile increased (see the circled portion of the curves in Fig. 2), implying that $n$ would be higher for aggressive drivers compared to normal drivers, and conservative drivers would have the lowest value of $n$. Therefore the level of aggressiveness of the driver and $T_{m}$ are negatively correlated, and $n$ and level of aggressiveness are positively correlated.

Table 1

Sign of correlation between various network features on the two-fluid model parameters $T_{m}$ and $n$ (a summary of findings from Ayadh, 1986; Ardekani et al., 1992; Bhat 1994).

\begin{tabular}{llc}
\hline Factors & $T_{m}$ & $n$ \\
\hline Signal density & + & - \\
Average speed limit & - & - \\
Fraction of approaches with signal progression & - & - \\
Average number of lanes per street & - & + \\
Fraction of one way streets & + & - \\
Fraction with actuated signals & & + \\
Average block lengths & & + \\
Average cycle length & - & \\
\hline
\end{tabular}




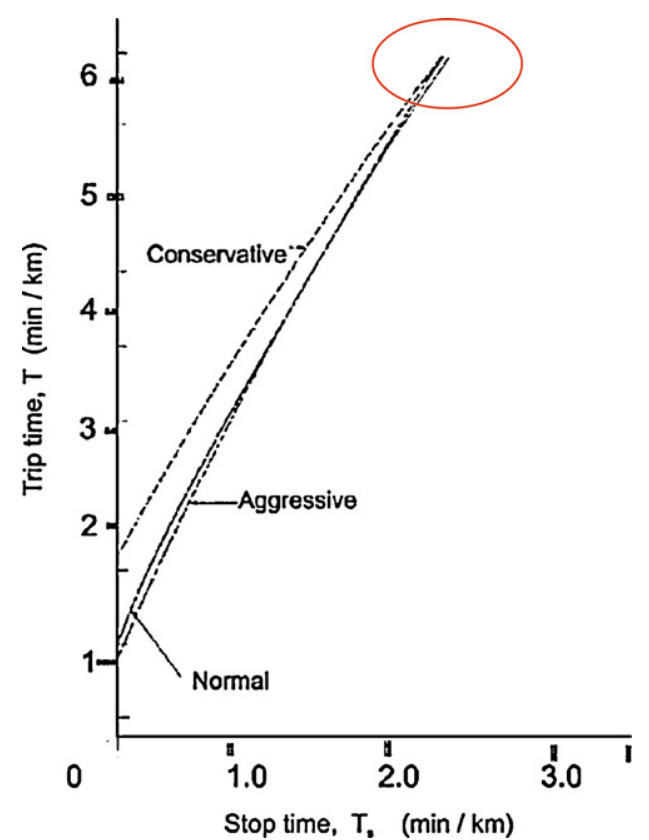

Fig. 2. The two fluid trends for aggressive, normal and conservative drivers in the Austin CBD (Herman et al., 1988).

These prior studies have clearly demonstrated that the two-fluid model is significantly affected by the environmental factors (Vo et al., 2007; Ayadh, 1986; Ardekani et al., 1992) and behavior of the drivers (Herman et al., 1988) on the network. Based on these studies, it may be argued that the estimate of the two-fluid model parameters for a network may lead to inference about the corridor characteristics and driver population on the roadway networks. Since, safety on arterial streets is also known to be significantly affected by driver behavior (Yan et al., 2008) and geometric and environmental factors (Abdel-Aty and Radwan, 2000); the parameters of the two-fluid model should be able to explain some of the crash patterns on the roadways. However, none of the studies have explicitly examined the relationship between safety on an arterial with the corresponding two-fluid model parameters. Relationship between the two-fluid model parameters and crash rates will provide a method to evaluate safety on a corridor and network level. If such a link does exist some of the crash patterns on the arterials may be anticipated in advance without having to collect crash data. In this regard, the two-fluid model parameters can work as surrogate measures of safety. This paper investigates the relationship between safety and the parameters of the two-fluid model for arterial streets in the Orlando (FL) metropolitan area.

\section{Chase-car methodology and data collection}

The two-fluid model parameters were estimated using the 20-25 observations for travel time per-mile and running time permile information collected from eight different arterial corridors in Orlando metropolitan area. Trip travel time data were collected on weekdays using the "chase car" methodology for the PM peak (16:30-18:00) during the months of April-2008 and May-2008. A one-mile travel segment was considered as a trip. Travel time and stop time were collected for multiple one-mile trips. In the chase-car method adopted in this study, the driver follows a randomly selected vehicle until it leaves the arterial, parks or the chase requires unsafe and/or illegal maneuvers, after which the next vehicle is selected for following. While transitioning from one vehicle to the next the vehicle is driven normally with respect to surrounding traffic. The two-fluid model estimated based on the per mile travel times recorded by the chase-car driver through this methodology is expected to be representative of the behavior of drivers of the corresponding arterial. An observer accompanied the driver on the data collection trips. The task of the observer includes recording the odometer readings, the absolute travel time per mile and stopped time per mile corresponding to the start and end of each trip. The chase car methodology employed in this study resulted in traveling between 20 and 25 miles on each of the eight arterials (This resulted in number of 20-25 observations equal to the number of 1-mile trips). The same methodology was also used by Jones and Farhat (2004).

\subsection{Arterial characteristics}

The set of eight arterials selected for the two-fluid model parameter estimation in this study had varying (but not widely varying) segment lengths. It was ensured that there were no significant changes in the characteristics of the corridors (such as number of lanes, speed limit) within the selected segment length. The arterials, section lengths as well as the average daily traffic on that section are shown in Table 2 .

Information on frequency and types of crashes was also collected for these particular stretches of the arterial roadways from the florida crash analysis and reporting (CAR) database. The inventory consists of crash data for various arterial corridors. Data regarding pavement conditions, number of lanes and access class for the particular corridor are also available in the roadway characteristics inventory $(\mathrm{RCI})$ database. Data regarding the number of signalized and unsignalized intersections (access points), leftturn bays, and two-way-left turning lanes were collected by driving around these corridors.

\section{Analysis and results}

\subsection{Two-fluid model parameter estimation}

According to the discussion provided earlier in this paper the relationship between per mile travel time and running time per mile is given by Eq. (4):

$T_{S}=T-T_{m}^{1 / n+1} T^{1 / n+1}$

Table 2

Description of arterials considered for this study.

\begin{tabular}{|c|c|c|c|c|}
\hline Number & Arterial street & Length (miles) & Average daily traffic (ADT) (vehicles) & Signal density (signals/mile) \\
\hline 1 & SR 426 & 4.59 & 37263 & 3.27 \\
\hline 2 & SR 50 between SR 434 and SR 436 & 6.53 & 48648 & 1.99 \\
\hline 3 & SR 50 East of SR 434 & 2.61 & 43599 & 3.07 \\
\hline 4 & SR 50 West of SR 436 & 5.84 & 47676 & 4.45 \\
\hline 5 & SR 434 & 4.13 & 48795 & 3.39 \\
\hline 6 & SR 436 (South section) & 6.05 & 51748 & 2.81 \\
\hline 7 & SR 436 (North Section) & 5.82 & 66460 & 3.09 \\
\hline 8 & SR 551 & 5.70 & 37731 & 1.75 \\
\hline
\end{tabular}




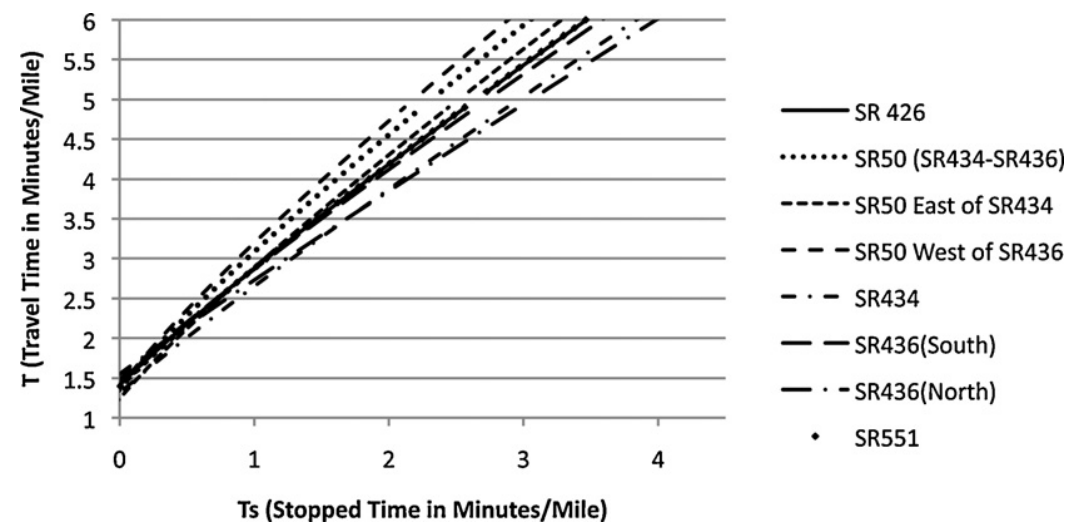

Fig. 3. The two-fluid model for each of the eight arterials.

Table 3

Parameter $T_{m}$ and $n$ of two-fluid model for different arterials.

\begin{tabular}{lllll}
\hline No. & Arterial street & $T_{m}$ & $n$ & $R^{2}$ \\
\hline 1 & SR 426 & 1.51 & 0.66 & 0.85 \\
2 & SR 50 between SR 434 and SR 436 & 1.36 & 1.09 & 0.77 \\
3 & SR 50 East of SR 434 & 1.24 & 0.99 & 0.76 \\
4 & SR 50 West of SR 436 & 1.38 & 1.24 & 0.56 \\
5 & SR 434 & 1.31 & 0.49 & 0.66 \\
6 & SR 436 (South section) & 1.48 & 0.54 & 0.45 \\
7 & SR 436 (North Section) & 1.55 & 0.24 & 0.45 \\
8 & SR 551 & 1.39 & 0.70 & 0.67 \\
\hline
\end{tabular}

Taking a logarithm of Eq. (4) on both sides, it is transformed into Eq. (5):

$\ln T_{r}=\frac{1}{n+1} \ln T_{m}+\frac{n}{n+1} \ln T$

$\ln T_{r}=B \ln T+A$

The coefficients $B$ and $A$ are estimated using linear regression between log of running time per mile and the log of travel time per mile for each of the arterial streets. The parameters $n$ and $T_{m}$ can then be estimated using Eqs. (7) and (8), respectively.

$n=\frac{B}{1-B}$

$T_{m}=e^{A / 1-B}$

The estimates of $n$ and $T_{m}$ obtained for each of the eight arterial segments using Eqs. (7) and (8) are listed in Table 3.

The table also provides the $R^{2}$ values observed for the linear regression model shown in Eq. (6). For the models described the $R^{2}$ fit were in an acceptable range, and therefore the parameters of the two-fluid model were used for further analyses. In the study conducted by Ardekani (1984) on the two-fluid models for various cities, a two-fluid model for San Antonio non CBD area was accepted with an $R^{2}$ of 0.45 , there have been other studies (e.g., Ardekani et al., 1992) in which two-fluid models with similar low values of
$R^{2}$ have been deemed satisfactory. The existence of the two-fluid model for these eight arterials replicates the results observed by Jones and Farhat (2004) on other arterial streets. It further demonstrates the applicability of the two-fluid approach on individual arterial streets.

\subsection{Comparing the arterials based on two-fluid model parameters}

The two-fluid models for the eight arterial have been plotted on the same graph in Fig. 3. Arterials with lower values of $T_{m}$ (i.e., lower intercepts on the $y$-axis in figure) have lower free-flow speeds when the stopped time is zero. In Fig. 3 curves corresponding to arterials having higher values of $n$ are steeper, suggesting that as the stopped time per mile increases the travel time per mile increases at a faster rate. It may also be observed that the curves corresponding to SR 426 and SR 551 are overlapping with each other. From Table 3 (and Fig. 3), it can be observed that SR 50 (East of SR 434) had the lowest free flow travel time per mile $\left(T_{m}=1.24 \mathrm{~min} / \mathrm{mile}\right)$, while SR 436 (North section) had the highest free flow travel time per mile $\left(T_{m}=1.55 \mathrm{~min} / \mathrm{mile}\right)$. It was found that SR 50 (West of SR 436) was found to have the highest value of $n$ (1.24), while SR 436 (North section) had the lowest value of $n(0.24)$.

The relationship between these parameters and operational performance of the corridor may be understood based on the discussion on essentials of the two-fluid model provided by Prigogine and Herman (1971) and Jones and Farhat (2004). For example, larger value of $T_{m}$ (i.e., free flow travel time) is indicative of the inefficient traffic control mechanism. The higher the value of $n$, the running time per mile increases at a faster rate for a unit increase in travel time per mile. This indicates that traffic on an arterial with a higher value of $n$ deteriorates at a faster rate. For instance operations on SR 50 (West of SR 436) deteriorates at a faster rate compared to the other seven arterials, this can be seen in Fig. 3, where at higher values of log travel time per mile the running time per mile is the highest on SR 50 (West of 436). In other words, lower value of ' $n$ ' indicates that interactions among vehicles

\section{Table 4}

Crash counts on each arterial based on type of crash.

\begin{tabular}{|c|c|c|c|c|c|c|}
\hline No. & Arterial street & Total crash & Rear-end crash & Angle crash & Sideswipe crash & Other crash \\
\hline 1 & SR 426 & 106 & 49 & 15 & 4 & 19 \\
\hline 2 & SR 50 between SR 434 and SR 436 & 342 & 152 & 60 & 21 & 28 \\
\hline 3 & SR 50 East of SR 434 & 116 & 63 & 15 & 4 & 15 \\
\hline 4 & SR 50 West of SR 436 & 382 & 173 & 61 & 26 & 35 \\
\hline 5 & SR434 & 201 & 87 & 39 & 13 & 10 \\
\hline 6 & SR436 (South section) & 346 & 139 & 57 & 14 & 65 \\
\hline 7 & SR436 (North Section) & 155 & 73 & 17 & 17 & 14 \\
\hline 8 & SR 551 & 203 & 82 & 44 & 13 & 7 \\
\hline
\end{tabular}


Table 5

Crash counts on each arterial based on level of severity.

\begin{tabular}{|c|c|c|c|c|c|c|}
\hline No. & Arterial street & No injury & Possible injury & Non-incapacitating injury & Incapacitating injury & Fatal injury \\
\hline 1 & SR 426 & 46 & 34 & 19 & 7 & 0 \\
\hline 2 & SR 50 between SR 434 and SR 436 & 140 & 101 & 73 & 16 & 10 \\
\hline 3 & SR 50 East of SR 434 & 43 & 35 & 24 & 6 & 3 \\
\hline 4 & SR 50 West of SR 436 & 141 & 130 & 81 & 22 & 2 \\
\hline 5 & SR 434 & 84 & 69 & 34 & 10 & 3 \\
\hline 6 & SR436 (South section) & 103 & 115 & 97 & 21 & 4 \\
\hline 7 & SR436 (North Section) & 77 & 35 & 33 & 8 & 1 \\
\hline 8 & SR 551 & 89 & 54 & 44 & 14 & 1 \\
\hline
\end{tabular}

increase slowly as congestion increases thereby making for a more efficient corridor (Prigogine and Herman, 1971; Jones and Farhat, 2004).

\subsection{Crash characteristics of arterial streets}

Frequency of crashes that occurred on these corridors in the year 2006 were extracted and classified by crash type. The information is summarized in Table 4 . The crashes were also classified based on the level of injury severity (Table 5).

It is important to note that the two-fluid model is an ergodic model that describes the relationship between travel time per mile and stopped time per mile. Therefore, it is akin to the 'fingerprint' of the corresponding arterial road. It describes the evolution of the travel time per mile as the stopped time per mile increases (congestion increases). Therefore, during a particular time of day when the congestion is high the stopped time per mile is high, and the travel time per mile can be determined from the two-fluid model and the stopped time per mile. Due to the ergodic nature of the model it describes the evolution of the travel time per mile as stopped time per mile increases regardless of the time of day. Therefore it would be valid to consider the entire year's crash data listed in Tables 4 and 5 with respect to the two-fluid model parameters.

\subsection{Relationship among crash characteristics, roadway characteristics, and two-fluid model parameters}

While the parameters' relationship with efficiency of the roadway network may be understood based on the discussion in the literature no one has ever explored their association with safety on the corresponding arterial streets. Therefore, the next step in this study was to examine crash, traffic, and geometric data for these arterials and their relationship with $n$ and $T_{m}$. The approach adopted for examining these relationships was to examine correlations between crash rates (crash counts normalized by vehicle miles traveled) and the two-fluid model parameters ( $n$ and $T_{m}$ ). Table 6 depicts the correlation coefficients between the two-fluid model parameters and various roadway features and crash rates on the arterials. Most of the geometric design and environmental information was collected from the database available from Florida Department of Transportation (FDOT), while some of the information such as access points and intersections per mile was collected manually driving around the arterial streets. The information manually collected for the streets has been marked with an asterisk in Table 6 . The significance of the correlation was tested based on the corresponding $p$-value; with $p$-value less than 0.15 indicating that the correlation coefficient is significantly different from zero.

The crash rates were calculated using the number of crashes belonging to each type (Table 4) as well as severity (Table 5) level. It is worth mentioning that the crashes involving incapacitating injury and fatal injury were combined together as "severe crashes" since the sample size for fatal crashes was too small.

In Table 6 all correlations found significant (based on the $p$-value threshold of 0.15 ) have been highlighted in grey. It is interesting to note that there is a negative correlation $(-0.56)$ between $T_{m}$ and $n$ at a moderately significant level. It essentially means that arterials with lower free flow travel time per mile $\left(T_{m}\right)$ (i.e., higher free flow speeds), proceed to congestion at a faster rate (higher value of $n$ ). Similar trends were observed by Herman et al. (1988). They found that with increase in aggressiveness of the driver the free-flow travel time per mile $\left(T_{m}\right)$ decreases and $n$ increases.

The average daily traffic per lane (ADTpL) had a significant negative correlation $(-0.70 ; p$-value $=0.05)$ with $n$ and a significant positive correlation $(+0.65 ; p$-value $=0.08)$ with $T_{m}$. This correlation can be explained by observing Eq. (9), which was derived from

Table 6

Correlation table (with coefficient and corresponding $p$-value) between parameters of two-fluid model and environmental as well as crash rates (crashes per million vehicle miles traveled).

\begin{tabular}{|c|c|c|c|}
\hline & $n$ & $T$ & $R^{2}$ \\
\hline \multirow[t]{2}{*}{$n$} & 1.00 & -0.57 & 0.40 \\
\hline & & 0.14 & 0.32 \\
\hline \multirow[t]{2}{*}{$T_{m}$} & -0.57 & 1.00 & -0.42 \\
\hline & 0.14 & & 0.30 \\
\hline \multirow[t]{2}{*}{ Speed limit } & -0.08 & -0.44 & -0.03 \\
\hline & 0.86 & 0.27 & 0.94 \\
\hline \multirow[t]{2}{*}{ Access management class } & 0.23 & 0.26 & -0.34 \\
\hline & 0.58 & 0.53 & 0.40 \\
\hline \multirow[t]{2}{*}{ Pavement conditions } & 0.13 & -0.27 & 0.35 \\
\hline & 0.77 & 0.52 & 0.39 \\
\hline \multirow[t]{2}{*}{ Number of lanes } & 0.01 & -0.02 & -0.59 \\
\hline & 0.98 & 0.96 & 0.12 \\
\hline \multirow[t]{2}{*}{ Access per mile ${ }^{a}$} & 0.42 & 0.17 & -0.45 \\
\hline & 0.31 & 0.69 & 0.27 \\
\hline \multirow[t]{2}{*}{ Length of two-way left turn per mile ${ }^{a}$} & 0.13 & 0.19 & -0.54 \\
\hline & 0.75 & 0.65 & 0.17 \\
\hline \multirow[t]{2}{*}{ Signals per mile ${ }^{a}$} & 0.16 & 0.02 & -0.22 \\
\hline & 0.70 & 0.96 & 0.59 \\
\hline \multirow[t]{2}{*}{ Average daily traffic } & -0.45 & 0.40 & -0.74 \\
\hline & 0.26 & 0.32 & 0.04 \\
\hline \multirow[t]{2}{*}{ Average daily traffic per lane } & -0.70 & 0.65 & -0.29 \\
\hline & 0.05 & 0.08 & 0.49 \\
\hline \multirow[t]{2}{*}{ Total crash rate } & 0.75 & -0.61 & -0.02 \\
\hline & 0.03 & 0.11 & 0.96 \\
\hline \multirow[t]{2}{*}{ Rear end crash rate } & 0.84 & -0.74 & 0.11 \\
\hline & 0.01 & 0.03 & 0.79 \\
\hline \multirow[t]{2}{*}{ Angle crash rate } & 0.56 & -0.55 & -0.01 \\
\hline & 0.15 & 0.16 & 0.99 \\
\hline \multirow[t]{2}{*}{ Sideswipe crash rate } & 0.50 & -0.30 & -0.28 \\
\hline & 0.20 & 0.47 & 0.51 \\
\hline \multirow[t]{2}{*}{ Unknown type crash rate } & 0.30 & 0.01 & -0.15 \\
\hline & 0.48 & 0.99 & 0.72 \\
\hline \multirow[t]{2}{*}{ No injury crash rate } & 0.79 & -0.71 & 0.19 \\
\hline & 0.02 & 0.05 & 0.65 \\
\hline \multirow[t]{2}{*}{ Possible injury crash rate } & 0.68 & -0.55 & -0.05 \\
\hline & 0.06 & 0.16 & 0.90 \\
\hline \multirow[t]{2}{*}{ Non-incapacitating injury crash rate } & 0.59 & -0.35 & -0.26 \\
\hline & 0.13 & 0.39 & 0.53 \\
\hline \multirow[t]{2}{*}{ Severe crash rate } & 0.76 & -0.68 & 0.09 \\
\hline & 0.03 & 0.06 & 0.84 \\
\hline
\end{tabular}

a Information collected manually by driving through the corresponding arterial streets. 
Eq. (1) (replace $v_{r}$ by $T_{r}$ and $v_{m}$ by $T_{m}$ ):

$f_{r}=\left(\frac{T_{m}}{T_{r}}\right)^{1 / n}$

Eq. (9) suggests that for given values of running time per mile and the parameter " $n$ "; higher the value of free flow travel time the higher will be the fraction of running vehicles. It explains the significant positive correlation between ADTpL (essentially a measure of traffic flow rate) and $T_{m}$. Also, for given values of running time per mile and free flow travel time per mile; a lower the value of $n$, would increase the fraction of running vehicles. It explains the significant negative correlation between ADTpL and $n$.

It is also observed that there is a significant negative correlation $(-0.74)$ between the average daily traffic (ADT) and the $R^{2}$ fit value for the linear regression model used to estimate the parameters of the two-fluid model. It implies that as ADT increases the $R^{2}$ fit decreases. A possible explanation is that as the operation on an arterial improves it approaches operational characteristics of a freeway. This results in violation of the ergodic assumption, thereby reducing the explanatory power (lower $R^{2}$ fit) of the two-fluid model. A similar high negative correlation $(-0.59)$ was observed between the number of lanes and the $R^{2}$ fit of the two-fluid model, this could be explained due to the high positive correlation $(+0.76)$ between number of lanes and ADT. It is expected since arterials with larger ADT are expanded to include more travel lanes. These results bring to light an important question: when does an arterial street stop being an arterial and starts behaving like a freeway?

No significant correlations were found between travel times, running times and the various types of crash rates or rates of crashes with different levels of severity. However, significant correlations were observed between the two-fluid parameters and the crash rates by types and levels of severity. It was found that $n$ and the rear-end crash rates were positively correlated $(+0.84)$ and that $T_{m}$ and the rear-end crash rates were negatively correlated $(-0.67)$.

To understand these correlations consider the partial differential of the running speed with respect to the fraction of stopped vehicles (replacing $f_{r}=1-f_{s}$ in Eq. (1) and differentiating with respect to $f_{s}$ ).

$$
\frac{d v_{r}}{d f_{s}}=-v_{m} n\left(1-f_{s}\right)^{n-1}
$$

From Eq. (10) it can be concluded that the rate of decrease in running speed with increase in fraction of stopped vehicles (LHS of Eq. (10)) increases with increase in $n$. It suggests that the congestion leads to higher reduction in running speeds on arterials that have higher values of $n$, in turn leading to conditions that may be more prone to rear-end collisions. This inference is possibly reflected in the positive correlation between $n$ and rear-end crash rate. Also, as observed earlier, $n$ and $T_{m}$ are negatively correlated, which possibly explains the negative correlation observed between rear-end crash rate and $T_{m}$. A positive correlation was also observed between angle crashes and the parameter $n$. But no correlation was found between the other types of crash rates and the two-fluid parameters. Note that the correlation coefficient and their significance do not represent causality but mere association. The interesting aspect of this study is to demonstrate how these correlations may actually be explained by traffic phenomenon observed on the roadways.

It is interesting to look at these results from the perspective of the findings from Herman et al. (1988). They found that aggressive drivers had lower values of $T_{m}$ and higher values of $n$, based on which higher rear-end crash rates can be attributed to aggressive behavior. The varying values of $n$ and $T_{m}$ on the eight arterial corridors possibly represent driver behavior on these roadways. The cause for the varying behavior could be geometric design of the arterials, or the differences in population of drivers during the peak hour or a combination of the two. Also, note that positive correla- tion between $n$ and total crash rate (0.75) and a negative correlation between $T_{m}$ and total crash rates $(-0.61)$ may be attributed to the high percentage of rear-end crashes (44\%) on these arterial streets.

Another interesting set of correlation coefficients were observed between $n$ and the rates of crashes with different injury severity. The correlation between $n$ and severe crash rate is positive and significant $(0.76 ; p$-value $=0.03)$. This indicates that an arterial corridor with higher value of $n$ has higher rate of severe crashes. Consequently, $T_{m}$ and severe crash rate were found to be negatively correlated $(-0.68)$. This could be due to the inherent negative correlation between $n$ and $T_{m}$, but it is also known that higher speeds (lower value of $T_{m}$ ) result in more severe crashes. It can be concluded that higher values of $n$ and lower values of $T_{m}$ are correlated with higher severe crash rates. Based on findings from Herman et al. (1988) higher values of $n$ and lower values of $T_{m}$ can be attributed to aggressive behavior, suggesting that aggressive behavior on certain arterials could be contributing to a higher rate of severe crashes. Once again it is not clear whether the aggressive behavior is due to environmental factors or the driving population or a combination of both. A significant positive correlation was observed between $n$ and no injury crash rash rate $(0.79 ; p$-value $=0.02)$ and a significant negative correlation was observed between $T_{m}$ and no injury crash rate $(-0.71 ; p$-value $=0.05)$. Similar correlation trends were observed between $n$ and possible injury crash rash rate (0.68) and between $T_{m}$ and possible injury crash rate $(-0.55)$. A positive correlation was also observed between non-incapacitating and $n(0.59)$. It is suspected that since a high percentage of no injury crashes (44\%) and possible injury crashes (55\%) are rear-end crashes, high correlations were observed between no-injury crash rate and possible injury crash rates with $n$ and $T_{m}$.

Based on the work by Ayadh (1986), Ardekani et al. (1992), and Bhat (1994) geometric factors like signal density, access density, the amount of two way left turning lanes per mile, speed limits may have been expected to show some associations with the parameters of the two-fluid model. In our analysis, however, we did not observe any significant correlations. It has an interesting implication, indicating that the correlations found between the two-fluid parameters and rear end crash rate as well as the crash rates for severe crashes may indeed be due to aggressive behavior attributable to the driving population using the road. It needs to be verified with further analysis.

\section{Conclusions}

This research studies the two-fluid models application to arterial streets. The model does not hold for freeways and therefore in its present form cannot be used to describe the behavior of drivers on uninterrupted flow facilities. The two-fluid model historically has been applied to evaluate the performance of urban networks as a whole (excluding the uninterrupted flow facilities). This study validates the preliminary explorations by Jones and Farhat (2004) and developed individual two-fluid model for eight arterial streets in the Orlando (FL) metropolitan area. More importantly, the relationship of two-fluid model parameters with geometric, crash, and aggregate traffic flow (e.g., ADT) characteristics of the corresponding corridors was also examined.

It was found that the $R^{2}$ fit of the two-fluid model deteriorates with higher ADT on the arterial streets. Higher ADT indicates that the roadway is able to serve vehicles at a faster rate. It in turn may be indicative of the fact that traffic operation on these roadways might be approaching traffic operation on freeways, possibly resulting in violation of the ergodic assumptions. There is a need to further investigate this phenomenon with data from more roadways.

It was also observed that the parameter " $n$ " was positively correlated with rear-end crash rate as well as rate of severe crashes. 
A negative correlation was observed between " $T_{m}$ " and rear-end crash rate as well as rate of severe crashes. Since none of the geometric factors were found to have a significant correlation with the two-fluid model parameters, it is suspected that the variation in two-fluid parameters between these arterials can be attributed to the varying aggressiveness of the driving behavior. It in turn may be resulting in higher rear-end as well as higher severe crash rate on arterials with more aggressive behavior. Rash or aggressive driving can be a consequence of skewed perceptions, which are observed among teenage and newly licensed drivers or drivers that tend to take more risks. Apart from risky attitudes and newly licensed drivers, factors such as distraction (for example, due to large number of signage on roadway) and frustration (due to long cycle lengths) can also result in aggressive responses from drivers. For example, a driver who is distracted and missed the light at a traffic signal turning from red to green would tend to accelerate suddenly as soon as he/she notices the green light.

The high correlations observed between the two-fluid parameters and rear-end crash rates as well as severe crash rate indicates that the two-fluid parameters have the potential as surrogate measures for evaluating safety of urban arterial corridors. Utilizing the two-fluid parameters as a surrogate could help in determining whether certain safety measures undertaken have helped improve safety without having to wait for sufficient crash data to be accumulated. However, this potential needs to be investigated further, with data for two-fluid models collected from multiple roadways from around the country. Improvements for corridors could be suggested based on the influence of roadway characteristics on the two-fluid model (Table 1), although further studies need to be undertaken to determine the influence of roadway characteristics on the two-fluid model.

\section{Acknowledgements}

The authors would like to thank the anonymous reviewers for their insightful comments that resulted in significantly improved paper.

\section{References}

Abdel-Aty, M.A., Radwan, A.E., 2000. Modeling traffic accident occurrence and involvement. Accident Analysis \& Prevention 32 (5), 633-642.

Ardekani, S.A., 1984. The two-fluid characterization of urban traffic: theory, observation, and experiment. PhD Dissertation, University of Texas at Austin.

Ardekani, S.A., Williams, J.C., Bhat, S., 1992. Influence of urban network features on quality of traffic service. Transportation Research Record, 6.

Ayadh, M.T., 1986. Influence of the City Geometric Features on the Two Fluid Model Parameters. Virginia Polytechnic Institute and State University.

Bhat, S.C.S. 1994. Effects of geometric and control features of network traffic: a simulation study. PhD Dissertation, University of Texas at Arlington.

Herman, R., Malakhoff, L., Ardekani, S., 1988. Trip time-stop time studies of extreme driver behaviors. Transportation research Part A: General 22 (6), 427-433.

Herman, R., Ardekani, S., 1984. Characterizing traffic conditions in urban areas. Transportation Science 18 (2), 101.

Jones, E.G., Farhat, W., 2004. Validation of two-fluid model of urban traffic for arterial streets. Transportation Research Record 1876, 132-141.

Mahmassani, H.S., Williams, J.C., Herman, R., 1984. Investigation of network-level traffic flow relationships: some simulation results. Transportation Research Record 971, 121-130.

Prigogine, I., Herman, R., 1971. Kinetic Theory of Vehicular Traffic. Elsevier Publishing Company.

Vo, P.T., Mattingly, S.P., Ardekani, S., Dilshad, Y., 2007. Comparison of quality of service in two central business districts: two-fluid model approach in Texas. Transportation Research Record 1999, 180-188.

Williams, J.C., Mahmassani, H.S., Herman, R., 1985. Analysis of traffic network flow relations and two-fluid model parameter sensitivity. Transportation Research Record 1005, 95-106.

Yan, X., Abdel-Aty, M., Radwan, E., Wang, X., Chilakapati, P., 2008. Validating a driving simulator using surrogate safety measures. Accident Analysis \& Prevention 40 (1), 274-288. 